\title{
Effective dispersion in a chemically heterogeneous medium under temporally fluctuating flow conditions
}

\author{
Vanessa Zavala-Sanchez, Marco Dentz *, Xavier Sanchez-Vila \\ Department of Geotechnical Engineering and Geosciences, Technical University of Catalonia (UPC), Barcelona, Spain
}

Received 10 August 2006; received in revised form 23 November 2006; accepted 27 November 2006

Available online 17 January 2007

\begin{abstract}
We investigate effective solute transport in a chemically heterogeneous medium subject to temporal fluctuations of the flow conditions. Focusing on spatial variations in the equilibrium adsorption properties, the corresponding fluctuating retardation factor is modeled as a stationary random space function. The temporal variability of the flow is represented by a stationary temporal random process. Solute spreading is quantified by effective dispersion coefficients, which are derived from the ensemble average of the second centered moments of the normalized solute distribution in a single disorder realization. Using first-order expansions in the variances of the respective random fields, we derive explicit compact expressions for the time behavior of the disorder induced contributions to the effective dispersion coefficients. Focusing on the contributions due to chemical heterogeneity and temporal fluctuations, we find enhanced transverse spreading characterized by a transverse effective dispersion coefficient that, in contrast to transport in steady flow fields, evolves to a disorder-induced macroscopic value (i.e., independent of local dispersion). At the same time, the asymptotic longitudinal dispersion coefficient can decrease. Under certain conditions the contribution to the longitudinal effective dispersion coefficient shows superdiffusive behavior, similar to that observed for transport in s stratified porous medium, before it decreases to its asymptotic value. The presented compact and easy to use expressions for the longitudinal and transverse effective dispersion coefficients can be used for the quantification of effective spreading and mixing in the context of the groundwater remediation based on hydraulic manipulation and for the effective modeling of reactive transport in heterogeneous media in general.
\end{abstract}

(C) 2006 Elsevier Ltd. All rights reserved.

Keywords: Reactive transport; Transverse dispersion; Temporal random flow; Random adsorption; Stochastic modeling; Effective dispersion

\section{Introduction}

The quantitative and qualitative understanding of transport in heterogeneous hydrogeochemical systems is of critical importance for transport modeling in natural groundwater systems, and as such a precondition for the analysis of groundwater contamination problems and for the design of soil and aquifer remediation strategies.

Local scale physical and chemical medium heterogeneities lead to an effective large scale transport behavior that

\footnotetext{
* Corresponding author.

E-mail addresses: vanessa.zavala@upc.edu(V.Zavala-Sanchez), marco. dentz@upc.edu (M. Dentz), xavier.sanchez-vila@upc.edu (X. SanchezVila).
}

is qualitatively and quantitatively different from the one observed in homogeneous media. The interaction of spatial fluctuations of the system parameters and local scale transport processes leads in general to enhanced solute spreading and mixing. The influence of spatially fluctuating physical and chemical system properties, such as hydraulic conductivity and sorption properties, for example, on solute transport has been studied extensively during the last two decades within the stochastic perturbative approach e.g. [1-6], among others. The later studies disregard the effects of local dispersion or focus on the asymptotic long time behavior of solute transport. The full temporal evolution of effective solute spreading in a chemically and physically heterogeneous medium for finite local dispersion has been studied in terms of the time behavior of effective 
dispersion coefficients, see e.g. [7-9]. The stochastic perturbative analysis yields an increase of the longitudinal effective dispersion coefficient to a macroscopic value as a result of physical and chemical medium heterogeneities, which is in agreement with experimental and numerical findings. The predicted asymptotic transverse dispersion coefficients, however, consistently underestimate numerical and experimental observations by at least one order of magnitude e.g. [10-12]. Recently, the scale dependence of macrodispersion and effective retardation factors for reactive chemicals have been studied in laboratory scale experiments [13] and critically compared to stochastic theories [14]. Lichtner and Tartakovsky [15] studied upscaled effective rate constants for heterogeneous reactions and their evolution with time, which are intimately connected to the observed scale dependence.

Transverse mixing is an important process for geochemical processes in rivers [16], stream-aquifer interactions [17], saltwater intrusion $[18,19]$, and microbial reactions $[20,21]$. The importance of transverse mixing for reactive transport modeling including precipitation (dissolution) reactions has recently been shown by [22]. As such, the correct quantification of transverse mixing and spreading is

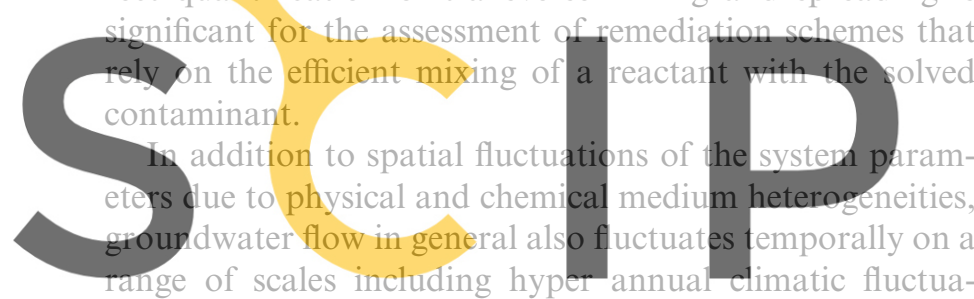
tions, seasonal and irrigation cycles and daily barometric

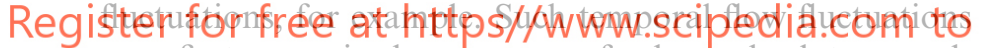
were first recognized as a source of enhanced solute spreading by Kinzelbach and Ackerer [23]. Rehfeldt and Gelhar [24] presented a stochastic approach for the quantification of the impact of temporal flow fluctuations in a physically heterogeneous medium. Within such a stochastic framework, Kabala and Sposito [25] studied macrodispersion for reactive transport in a spatially heterogeneous medium. Solute spreading in a heterogeneous medium for periodic (deterministic) time fluctuations of the hydraulic gradient for purely advective transport (vanishing local dispersion) has been analyzed by, e.g., Zhang and Neuman [26] and Dagan et al. [27]. Recently, Cirpka [28] studied the enhancement of transverse dispersion of kinetically sorbing compounds in spatially uniform flow field under sinusoidal (deterministic) temporal fluctuations and vanishing local dispersion.

A recent approach to characterize and quantify effective spreading and mixing in time-fluctuating flow through a physically heterogeneous medium is an analysis in terms of effective dispersion coefficients [29-31]. Effective dispersion coefficients characterize effective solute spreading and mixing in an heterogeneous environment [7,8,32]. For transport in time fluctuating spatial random flow fields, it was shown that the interaction between temporal fluctuations, local dispersion and spatial heterogeneity leads to macroscopic contributions to the longitudinal as well as, and more importantly, the transverse effective dispersion coefficients [29-31].

Here we study the impact of the interaction of local dispersion, chemical heterogeneity and temporal fluctuations of the flow conditions on the effective transport behavior of a sorbing solute. We focus on linear sorption reactions under instantaneous local equilibrium conditions. In a chemical heterogeneous medium, the local sorption properties are subject to spatial fluctuations, which can be characterized by a spatially varying retardation coefficient. The effective transport behavior in this practically relevant scenario is studied within a stochastic perturbative approach. Within this approach we derive compact analytical expressions for the temporal evolution of the longitudinal and transverse effective dispersion coefficients.

\section{Basics}

The objective is to quantify effective solute spreading and mixing of a sorbing chemical in terms of effective transport coefficients. To this end, in the following we define the

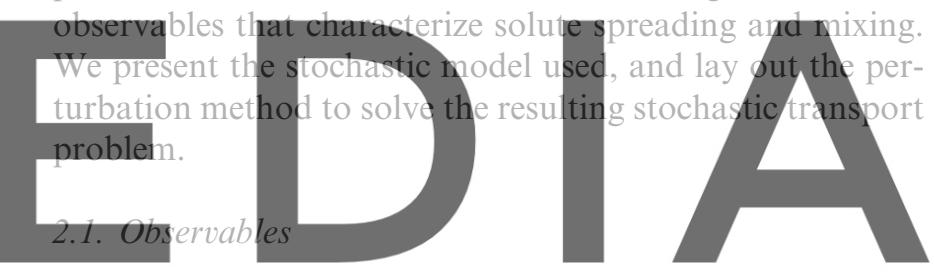

The total concentration distribution of the sorbing

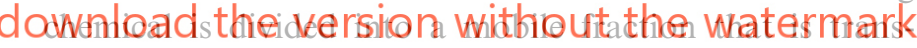
ported in the flow through the medium, and a fraction that is adsorbed to the solid matrix,

$c(\mathbf{x}, t)=\phi(\mathbf{x}) c_{\mathrm{m}}(\mathbf{x}, t)+[1-\phi(\mathbf{x})] c_{\mathrm{ad}}(\mathbf{x}, t)$,

where $c_{\mathrm{m}}(x, t)$, and $c_{\mathrm{ad}}(x, t)$ denote the spatial distributions of the mobile and adsorbed concentrations, respectively; and $\phi(x)$ denotes porosity.

As the simplest measures for the analysis of the evolution of the sorbing chemical, we study the effective center of mass velocity and effective dispersion coefficients of the (normalized) distribution density of mobile solute fraction [7],

$p(\mathbf{x}, t)=\frac{c_{\mathrm{m}}(\mathbf{x}, t)}{\int \mathrm{d}^{d} y c_{\mathrm{m}}(\mathbf{y}, t)}$.

In a given aquifer the center of mass velocity and dispersion coefficients are defined by

$u_{j}(t)=\frac{\mathrm{d}}{\mathrm{d} t} m_{j}^{(1)}(t)$,

$D_{i j}(t)=\frac{1}{2} \frac{\mathrm{d}}{\mathrm{d} t}\left[m_{i j}(t)^{(2)}-m_{i}^{(1)}(t) m_{j}^{(1)}(t)\right]$,

where $m_{i}^{(1)}(t)$ and $m_{i j}^{(2)}(t)$ are the first and second moments of the normalized spatial concentration distribution, defined as 
$m_{i}^{(1)}(t)=\int \mathrm{d}^{d} x x_{i} p(\mathbf{x}, t)$

$m_{i j}^{(2)}(t)=\int \mathrm{d}^{d} x x_{i} x_{j} p(\mathbf{x}, t)$.

As detailed in Section 2.3, in a stochastic analysis, the spatially heterogeneous aquifer is identified with one particular realization of an ensemble of aquifer realizations, while the temporally fluctuating groundwater flow is modeled by a correlated stationary random process. In such an approach, the effective transport coefficients can be expressed as averages over the ensemble of all possible realizations of the spatial and temporal random processes. The effective transport velocity and dispersion coefficients are then defined as the ensemble averages over their local scale counternarts (3) and (4), resnectively, $[29,311$

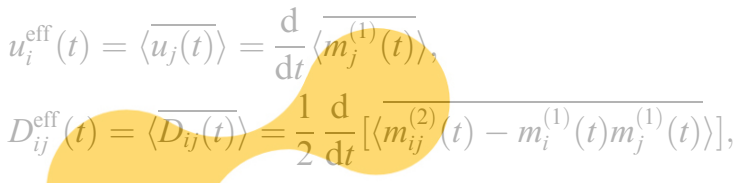

where the overbar denotes the average over the spatial random field, the angular brackets denote the average over the temporal random process. The effective dispersion coeffi-

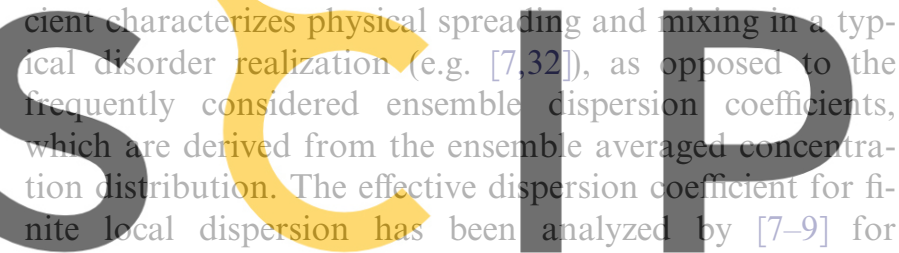
transport in a physically and chemically heterogeneous medium under steady flow conditions, and [29-31] for pas-

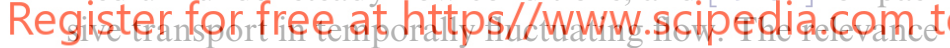
of effective dispersion coefficients for the quantification of solute mixing and thus for reactive transport modeling has been outlined by [33-35]. Thus, here we focus exclusively on the analysis of the effective transport velocity and dispersion coefficients.

\subsection{Transport model}

The temporal evolution of the mobile concentration $c_{\mathrm{m}}(\mathbf{x}, t)$ under spatially varying equilibrium sorption properties can be described by (e.g. [7]),

$$
\begin{aligned}
& R(\mathbf{x}) \frac{\partial c_{\mathrm{m}}(\mathbf{x}, t)}{\partial t}+\mathbf{q}(\mathbf{x}, t) \cdot \nabla c_{\mathrm{m}}(\mathbf{x}, t)-\nabla \mathbf{D}_{0} \nabla c_{\mathrm{m}}(\mathbf{x}, t) \\
& \quad=\rho(\mathbf{x}) \delta(t),
\end{aligned}
$$

where the retardation coefficient is defined by [7],

$R(\mathbf{x}) \equiv \phi(\mathbf{x})+[1-\phi(\mathbf{x})] k_{\mathrm{d}}(\mathbf{x})$,

with a positive spatially varying distribution coefficient $k_{\mathrm{d}}(\boldsymbol{x})$. Note that for technical convenience $t \in(-\infty,+\infty)$, see e.g. [7].

The (constant) local dispersion tensor $D_{0}$ is assumed to be diagonal, i.e., $D_{i j}=\delta_{i j} D_{i j} ; \mathbf{q}(\mathbf{x}, t)$ is the divergence-free spatio-temporally fluctuating Darcy velocity (e.g. [24,29]).
The right side of (9) represents the initial condition for an instantaneous solute injection at $t=0, c_{\mathrm{m}}(\mathbf{x}, t=0)=\rho(\mathbf{x})$. This implies for the mobile concentration $c_{\mathrm{m}}(\mathbf{x}, t=0)=$ $R(\mathbf{x})^{-1} \rho(\mathbf{x})$.

Here we study transport for a solute evolving from a point-like injection at $t=0$, i.e., $\rho(\mathbf{x})=\delta(\mathbf{x})$. We assume vanishing concentration at the boundaries at infinity.

\subsection{Stochastic model}

The spatially fluctuating retardation factor $R(\mathbf{x})$ and the hydraulic conductivity $K(\mathbf{x})$ here are modeled as stationary spatial random fields, while the temporal fluctuations of the flow boundary conditions that induce a fluctuating mean hydraulic gradient are modeled as a stationary temporal random process (e.g. [24,29])

We split the retardation factor into its mean value and fluctuations about it

\section{$R(\mathbf{x})=\bar{R}[1-\mu(\mathbf{x})]$}

where $\bar{R}$ is the ensemble averaged retardation factor, $\mu(\mathbf{x})$ denotes the normalized fluctuation, whose ensemble average is zero by definition, $\mu(\mathbf{x})=0$. The correlation function

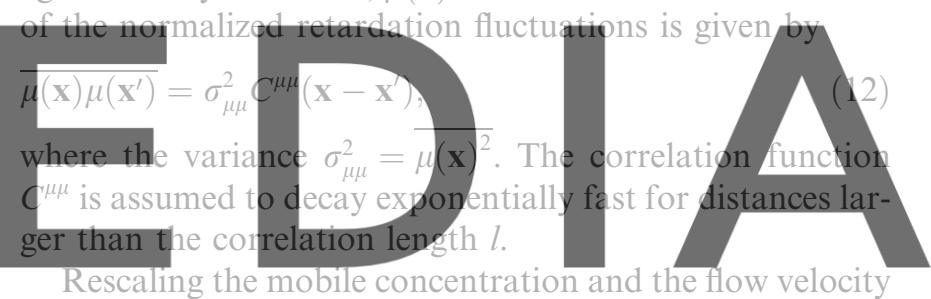

Rescaling the mobile concentration ancthe fow velocity as well as the local dispersion coefficients by mean retardadowabaach the version without the watermark
$g(\mathbf{x}, t)=\bar{R} c_{\mathrm{m}}(\mathbf{x}, t)$
$\mathrm{D}=\frac{\mathrm{D}_{0}}{\bar{R}}$
$\mathbf{u}(\mathbf{x}, t)=\frac{q(\mathbf{x}, t)}{\bar{R}}$

we obtain from (9) a transport equation for $g(\mathbf{x}, t)$,

$$
\begin{aligned}
& \frac{\partial g(\mathbf{x}, t)}{\partial t}+\mathbf{u}(\mathbf{x}, t) \cdot \nabla g(\mathbf{x}, t)-\nabla \cdot \mathbf{D} \nabla g(\mathbf{x}, t) \\
& \quad=\delta(\mathbf{x}) \delta(t)+\mu(\mathbf{x}) \frac{\partial g(\mathbf{x}, t)}{\partial t}
\end{aligned}
$$

Note that the normalized mobile concentration (2) reads in terms of the rescaled mobile concentration $g(\mathbf{x}, t)$ as,

$p(\mathbf{x}, t)=\frac{g(\mathbf{x}, t)}{\int \mathrm{d}^{d} y g(\mathbf{y}, t)}$.

For quasi-steady flow conditions, i.e., instantaneous propagation of a temporal change in the flow boundary conditions, the normalized first-order solution of the flow problem (in the fluctuations of the log-hydraulic conductivity) can be decomposed as (e.g. [24,29])

$\mathbf{u}(\mathbf{x}, t)=\mathbf{u}(t)-\mathbf{u}^{\prime}(\mathbf{x}, t)$.

The mean flow direction is aligned with the 1-direction of the coordinate system

$\langle\overline{\mathbf{u}(\mathbf{x}, t)}\rangle=u \mathbf{e}_{1}$ 
with $\mathbf{e}_{1}$ the unit vector in 1-direction. The purely timedependent part is given by

$\mathbf{u}(t)=u\left[\mathbf{e}_{1}-\mathbf{v}(t)\right]$

with the normalized purely temporal velocity fluctuations $\boldsymbol{v}(t)$, whose mean is zero by definition,

$\langle\boldsymbol{v}(t)\rangle=\left\langle\overline{\mathbf{u}^{\prime}(\mathbf{x}, t)}\right\rangle \equiv 0$.

A brief discussion of the quality of the approximation of quasi-steady flow is given in [31]. The correlation functions of the normalized temporal velocity fluctuations $v(t)$ are given by

$\left\langle v_{i}(t) v_{j}\left(t^{\prime}\right)\right\rangle=\sigma_{v v}^{2} C_{i j}^{v v}\left(t-t^{\prime}\right)$

with the variance $\sigma_{v v}^{2}$ of the temporal fluctuations, which, for simplicity, is assumed to be equal in all directions. The correlations of the $v_{i}(t)$ are assumed to be short range, i.e., to decrease quickly for times larger than the correlation time $\tau$.

Using decomposition (16) in (14), we obtain our working equation

$\frac{\partial g(\mathbf{x}, t)}{\partial t}+\mathbf{u}(t) \cdot \nabla g(\mathbf{x}, t)-\nabla \mathbf{D} \nabla g(\mathbf{x}, t) \rho(\mathbf{x}) \delta(t)$
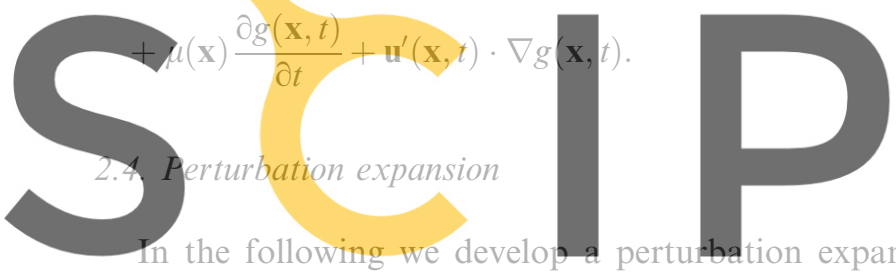

for $g(\mathbf{x}, t)$ up to first-order in the variances of the random

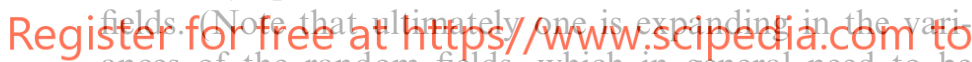
ances of the random fields, which in general need to be small for the perturbation results to be meaningful). This expansion serves as the basis for corresponding expansions for the effective transport velocity and dispersion coefficients.

\subsubsection{Solute distribution}

We reformulate the transport equation (21) in terms of an equivalent integral equation in Fourier space, from which we derive a perturbation solution of the transport problem. The Fourier transform $\tilde{g}(\mathbf{k}, t)$ of $g(\mathbf{x}, t)$ here is defined by

$\tilde{g}(\mathbf{k}, t)=\int \mathrm{d}^{d} x g(\mathbf{x}, t) \exp (\mathrm{ik} \cdot \mathbf{x})$,

$g(\mathbf{x}, t)=\int_{k} \tilde{g}(\mathbf{k}, t) \exp (-\mathrm{ik} \cdot \mathbf{x})$

where Fourier-transformed quantities are marked by a tilde. Here and in the following we employ the short-hand notation,

$\int_{k} \cdots=\int \frac{\mathrm{d}^{d} k}{(2 \pi)^{d}} \cdots$

The integral equation for $\tilde{g}(\mathbf{k}, t)$ is given by (see e.g. [29,31])

$$
\begin{aligned}
\tilde{g}(\mathbf{k}, t)= & \tilde{g}_{0}(\mathbf{k}, t, 0) \tilde{\rho}(\mathbf{k}) \\
& +\int_{k^{\prime}} \int_{-\infty}^{\infty} \mathrm{d} t^{\prime} \tilde{g}_{0}\left(\mathbf{k}, t, t^{\prime}\right) \mathbf{L}\left(\mathbf{k}, \mathbf{k}^{\prime}, t^{\prime}\right) \tilde{g}\left(\mathbf{k}-\mathbf{k}^{\prime}, t^{\prime}\right),
\end{aligned}
$$

with the perturbation operator defined by,

$\mathbf{L}\left(\mathbf{k}, \mathbf{k}^{\prime}, t\right) \equiv \tilde{\mu}\left(\mathbf{k}^{\prime}\right) \frac{\partial}{\partial t}-\mathrm{i} \mathbf{k} \tilde{\mathbf{u}}^{\prime}\left(\mathbf{k}^{\prime}, t^{\prime}\right)$.

We used here the incompressibility condition $\mathbf{k} \cdot \tilde{\mathbf{u}}(\mathbf{k}, t)=0$. The Fourier-transformed Green function of the transport problem (21) for $\mu(\mathbf{x})=0$ and $\mathbf{u}^{\prime}(\mathbf{x}, t)=\mathbf{0}$, denoted in (25) by $\tilde{g}_{0}\left(\mathbf{k}, t, t^{\prime}\right)$ reads as (e.g. [31]),

$\tilde{g}_{0}\left(\mathbf{k}, t, t^{\prime}\right)=\exp \left[-\mathbf{k D k}\left(t-t^{\prime}\right)+\mathrm{ik} \cdot \int_{t^{\prime}}^{t} \mathrm{~d} \tau \mathbf{u}(\tau)\right] \Theta\left(t-t^{\prime}\right)$

with $\Theta(t)$ the Heaviside step function as defined in [36].

Iteration of the integral equation (25) yields a perturbation series for $\tilde{g}(\mathbf{k}, t)$ in terms of the perturbation operator $\mathbf{L}\left(\mathbf{k}, \mathbf{k}^{\prime}, t\right)$,

$\tilde{g}(\mathbf{k}, t)=\tilde{g}_{0}(\mathbf{k}, t, 0) \tilde{\rho}(\mathbf{k})+\int_{k^{\prime}} \int_{-\infty}^{\infty} \mathrm{d} t^{\prime} \tilde{g}_{0}\left(\mathbf{k}, t, t^{\prime}\right) \mathbf{L}\left(\mathbf{k}, \mathbf{k}^{\prime}, t^{\prime}\right)$ $\begin{aligned} & \times \tilde{g}_{0}\left(\mathbf{k}-\mathbf{k}^{\prime}, t^{\prime}, Q\right)+\int_{k^{\prime}} \int_{-\infty}^{\infty} \mathrm{d} t^{\prime} \int_{k^{\prime \prime}} \int_{-\infty}^{\infty} d t^{\prime \prime} \tilde{g}_{0}\left(\mathbf{k} t, t^{\prime}\right) \\ & \times \mathbb{L}\left(\mathbf{k}, \mathbf{k}^{\prime}, t^{\prime}\right) \tilde{g}_{0}\left(\mathbf{k}-\mathbf{k}^{\prime}, t^{\prime}, t^{\prime \prime}\right) \times \mathbb{L}\left(\mathbf{k}-\mathbf{l}, \mathbf{k}^{\prime \prime}, t^{\prime \prime}\right) \\ & \times \tilde{g}_{0}\left(\mathbf{k}-\mathbf{k}^{\prime \prime}-\mathbf{k}^{\prime \prime}, t^{\prime \prime}, 0\right)+\cdots\end{aligned}$ constitutes the basis for the following perturbation dowanyejad the version without the watermark Note that the Green function $\tilde{g}_{0}\left(\mathbf{k}, t, t^{\prime}\right)$, (27), depends on the temporal fluctuations $v_{i}(t)$. To obtain consistent expressions for the transport coefficients, $\tilde{g}_{0}\left(\mathbf{k}, t, t^{\prime}\right)$ will be expanded in powers of $v(t)$ in the following (e.g. [29,31])

$\tilde{g}_{0}\left(\mathbf{k}, t, t^{\prime}\right)=\tilde{c_{0}}\left(\mathbf{k}, t-t^{\prime}\right)\left[1+u \mathbf{i k} \cdot \int_{t^{\prime}}^{t} \mathrm{~d} t^{\prime \prime} \boldsymbol{v}\left(t^{\prime \prime}\right)+\cdots\right] \Theta\left(t-t^{\prime}\right)$.

We defined here

$\tilde{c_{0}}(\mathbf{k}, t)=\exp \left(-\mathbf{k D k} t+\mathrm{i} k_{1} u t\right)$,

which is the Fourier transform of the solution of (21) for $\boldsymbol{v}(t)=\mathbf{u}^{\prime}(\mathbf{x}, t) \equiv \mathbf{0}$, and $\mu(\mathbf{x})=0$.

\subsubsection{Transport coefficients}

The effective transport velocity $u_{i}^{\text {eff }}(t),(7)$, and the dispersion coefficients $D_{i j}^{\text {eff }}(t),(8)$, can be expressed in terms of the Fourier transform of $p(\mathbf{x}, t)$ as (e.g. [7,8])

$u_{i}^{\text {eff }}(t)=\left.\frac{1}{\mathrm{i}} \frac{\mathrm{d}}{\mathrm{d} t} \frac{\partial}{\partial k_{i}}\langle\overline{\ln \tilde{p}(\mathbf{k}, t)}\rangle\right|_{\mathbf{k}=\mathbf{0}}$
$D_{i j}^{\text {eff }}(t)=-\left.\frac{1}{2} \frac{\mathrm{d}}{\mathrm{d} t} \frac{\partial}{\partial k_{i}} \frac{\partial}{\partial k_{j}}\langle\overline{\ln \tilde{p}(\mathbf{k}, t)}\rangle\right|_{\mathbf{k}=\mathbf{0}}$

with the Fourier transform of $p(\mathbf{x}, t)$ given by 
$\tilde{p}(\mathbf{k}, t)=\frac{\tilde{g}(\mathbf{k}, t)}{\tilde{g}(0, t)}$.

Relations (31) and (32) can be readily verified by using the definition of the Fourier transform (23).

Inserting (28) into (32) and expanding the resulting expression for small variances of the random fields the dispersion coefficients decompose into,

$D_{i j}^{\mathrm{eff}}(t)=D_{i j}+\delta^{\mu \mu} D_{i j}^{\mathrm{eff}}(t)+\delta^{u u} D_{i j}^{\mathrm{eff}}(t)+\delta^{u \mu} D_{i j}^{\mathrm{eff}}(t)+\delta^{\mu v} D_{i j}^{\mathrm{eff}}(t)$

and accordingly for the effective center of mass velocity (31). Note that, strictly speaking, for such an expansion to be valid, the variances of the random fields are required to be small. The validity of such a simultaneous expansion in the variances of the spatial and temporal random fields has been discussed in [31].

The effective dispersion coefficient $D_{i j}^{\text {eff }}(t)$ is given by the sum of the contributions due to (i) local dispersion $D_{i j}$, (ii) chemical heterogeneity $\delta^{\mu \mu} D_{i j}^{\text {eff }}$ (see [7]), (iii) physical heterogeneity and temporal fluctuations of the flow conditions $\delta^{u u} D_{i j}^{\text {eff }}$ (see e.g. $[8,9,29,31]$ ), (iv) cross-correlations between physical and chemical heterogeneity $\delta^{u \mu} D_{i j}^{\text {eff }}$ (see e.g. [8] for

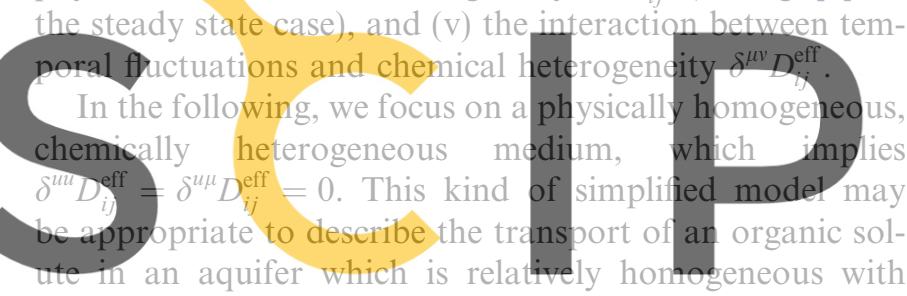
respect to the hydraulic conductivity, but has a strongly

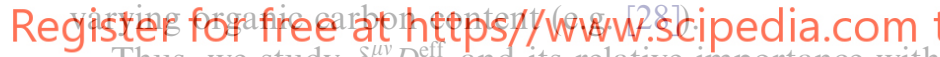
Thus, we study $\delta^{\mu \nu} D_{i j}^{\text {eff }}$ and its relative importance with respect to the contribution $\delta^{\mu \mu} D_{i j}^{\mathrm{eff}}$ due to chemical heterogeneity only [7]. Explicit expressions for $\delta^{\mu \nu} u_{i}^{\mathrm{eff}}$ and $\delta^{\mu \nu} D_{i j}^{\mathrm{eff}}$ are given in Appendix A.

\subsubsection{Time scales and effective parameters}

The temporal evolution of the effective dispersion coefficients is determined by three characteristic time scales, $\tau_{u}, \tau_{D_{i}}$, and $\tau_{\kappa}$. The advection time scale $\tau_{u}$ measures the time for the solute to be advected over one longitudinal correlation length $l_{1}$,

$\tau_{u}=\frac{l_{1}}{u}$.

The dispersion time scales $\tau_{D_{i}}$ characterize the time for dispersive transport over one correlation length $l_{i}$,

$\tau_{D_{i}}=\frac{l_{i}^{2}}{D_{i i}}$.

The Kubo time scale $\tau_{\kappa}$ [31], measures the time for the local dispersive spreading over an effective length that is given by the correlation length $l$ and the Kubo length $l_{\kappa}=u \tau$,

$\tau_{\kappa}=\left(1+\kappa^{2}\right) \tau_{D_{1}}=\left(l^{2}+l_{\kappa}^{2}\right) \tau_{D_{1}}$.
We defined here the non-dimensional Kubo number, $\kappa=\tau / \tau_{u}=l_{\kappa} / l_{1}$, which compares the correlation time $\tau$ to the advection time scale $\tau_{u}$, and equivalently the Kubo distance $l_{\kappa}=u \tau$ (which denotes the length over which the solute is advected by the mean flow during one correlation time $\tau$ ) to the correlation length in direction of the mean flow $l_{1}$.

The non-dimensional Peclet numbers $P e_{i}=\tau_{D_{i}} / \tau_{u}$ compare the strength of advective and dispersive transport mechanism. In many hydrological applications transport is advection dominated, which implies large Peclet numbers, $P e \gg 1$, or accordingly, small inverse Peclet numbers $\epsilon_{i} \equiv \tau_{u} / \tau_{D_{i}}=D_{i i} l_{1} /\left(u l_{i}^{2}\right)$. In the following we will develop simple compact expressions for the effective dispersion coefficients under the assumption of small $\epsilon_{i}$.

Note that for times smaller than the advection timescale. $t \leqslant \tau_{u}$, the solute has moved by mean advection over a distance shorter than the correlation length $l_{1}$ of the medium, and has spread by local dispersion over a distance which is much smaller than the corresponding correlation distance. On such short scales the medium looks quasi homogeneous and the solute does not "see" the heterogeneity of the medium. Thus, the spatial ensemble average and accordingly

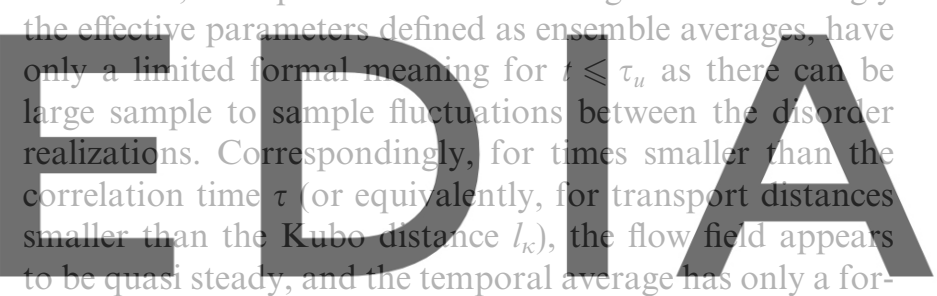

mal meaning. The impact of spatial heterogeneity and tem-

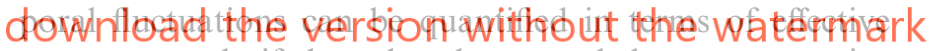
parameter only if the solute has sampled a representative part of the spectrum of spatio-temporal variability.

Appendix A summarizes the somewhat lengthy calculations that lead to the expressions for the effective center of mass velocity and dispersion coefficients presented in the following. We employ an expansion for small inverse Peclet numbers $\epsilon_{i} \ll 1$ and time large compared to the advection time scale $t \gg \tau_{u}$ (e.g. [31]) in order to simplify the lengthy expressions given in Appendix A.

2.4.3.1. Effective center of mass velocity. The leading contributions for small $\epsilon_{i}$ to the effective center of mass velocity, $\delta^{\mu v} u_{i}^{\text {eff }}$, are given by (see Appendix A),

$$
\begin{aligned}
\delta^{\mu v} u_{i}^{\mathrm{eff}}(t)= & u \sigma_{\mu \mu}^{2} \sigma_{v v}^{2} \int_{k^{\prime}} \tilde{C}^{\mu \mu}\left(\mathbf{k}^{\prime}\right)\left[\delta_{i 1} A\left(\mathbf{k}^{\prime}, t\right)+A_{i}\left(\mathbf{k}^{\prime}, t, 0\right)\right] \\
& \times \exp \left(-\mathrm{i} u k_{1}^{\prime} t\right)+\cdots,
\end{aligned}
$$

where the dots denote subleading contributions of the order of the inverse Peclet numbers. The auxiliary functions $A(\mathbf{k}, t)$ and $A_{i}\left(\mathbf{k}^{\prime}, t, 0\right)$ are defined by (A.18) and (A.19) in Appendix A. For short-range correlation functions, expression (38) decreases exponentially fast on the advection time scale $\tau_{u}$. 
2.4.3.2. Effective dispersion coefficients. As outlined in Appendix A, the leading behavior of $\delta^{\mu v} D_{i j}^{\text {eff }}(t)$ for small inverse Peclet numbers is given by,

$$
\begin{aligned}
\delta^{\mu v} D_{i j}^{\mathrm{eff}}(t)= & u^{2} \sigma_{\mu \mu}^{2} \sigma_{v v}^{2} \int_{k^{\prime}} \int_{0}^{t} \mathrm{~d} t^{\prime} \tilde{C}^{\mu \mu}\left(\mathbf{k}^{\prime}\right)\left[\delta_{i 1} \delta_{j 1} A\left(\mathbf{k}^{\prime}, t^{\prime}\right)\right. \\
& \left.+\delta_{j 1} A_{i}\left(\mathbf{k}^{\prime}, t^{\prime}, 0\right)+\delta_{i 1} A_{j}\left(\mathbf{k}^{\prime}, t^{\prime}, 0\right)+C_{i j}^{v v}\left(t^{\prime}\right)\right] \\
& \times \exp \left(-\mathrm{i} u k_{1}^{\prime} t^{\prime}\right)\left[1-\exp \left(-2 k_{j}^{\prime 2} l_{j}^{2} t / \tau_{D_{j}}\right)\right] .
\end{aligned}
$$

\section{Explicit expressions for the effective dispersion coefficients}

We focus on a transport situation for which the temporal fluctuations are transverse to the direction of the mean flow velocity, $\boldsymbol{v}(t)=\left[0, v_{2}(t), \ldots, v_{\mathrm{d}}(t)\right]^{\mathrm{T}}$. In this case, (39) simplifies to,

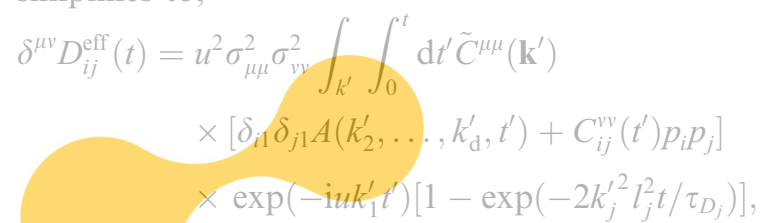

where we defined $p_{i}=\left(1-\delta_{i 1}\right)$. In the following, we study without loss of generality fluctuations in 2-direction, i.e.,

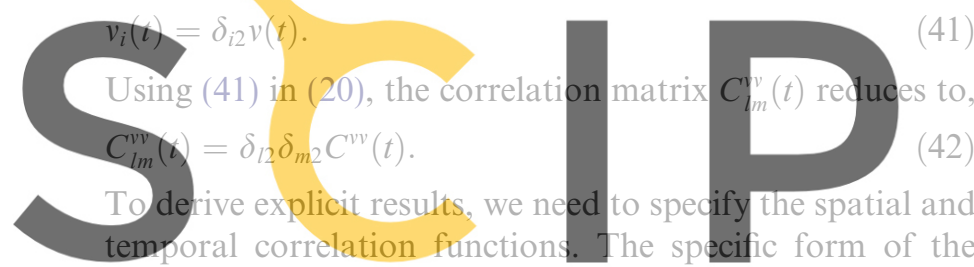
spatial and temporal correlation functions $C^{\mu \mu}$ and $C^{v v}$ is Regist some extent arbitrary. A convenient choice made in Ste rited fure are Gattos
fluctuations of the flow field are assumed to be Gaussian correlated (e.g. [31]), i.e.,

$C^{v v}(t)=\exp \left[-\frac{t^{2}}{2 \tau^{2}}\right]$,

with $\tau$ the correlation time. In analogy to [7], we use a Gaussian shaped correlation function for the retardation field $\mu$, which in Fourier space reads as,

$C^{\mu \mu}(\mathbf{k})=(2 \pi)^{\frac{d}{2}} \prod_{i=1}^{d} l_{i} \exp \left[-\frac{1}{2}\left(k_{i} l_{i}\right)^{2}\right]$.

The length scales $l_{i}$ are the correlation lengths of the retardation fields in direction $i$ (with $i=1, \ldots, d$ ).

Inserting (42) with (43) and (44) into (40) and using (A.18) for $A\left(k_{2}^{\prime}, t^{\prime}\right)$, we obtain,

$$
\begin{aligned}
\delta^{\mu v} D_{i i}^{\text {eff }}(t)= & u l_{1} \sigma_{\mu \mu}^{2} \sigma_{v v}^{2} \int_{k^{\prime}} \int_{0}^{t / \tau_{u}} \mathrm{~d} t^{\prime}\left[\delta_{i 1} \frac{l_{1}^{2}}{l_{2}^{2}} \frac{k_{2}^{\prime 2}}{2} \int_{0}^{t^{\prime}} \mathrm{d} y \int_{0}^{t^{\prime}} \mathrm{d} y^{\prime}\right. \\
& \left.\times \exp \left[-\frac{\left(y-y^{\prime}\right)^{2}}{2 \kappa^{2}}\right]+\delta_{i 2} \exp \left(-\frac{t^{\prime^{2}}}{2 \kappa^{2}}\right)\right] \\
& \times \exp \left(-\mathrm{i} k_{1}^{\prime} t^{\prime}\right)\left\{1-\exp \left[-\frac{k_{j}^{\prime 2}}{2}\left(1+4 t / \tau_{D_{j}}\right)\right]\right\} .
\end{aligned}
$$

In the following we will restrict ourselves to isotropic disorder scenario, i.e., $l_{1}=\cdots=l_{\mathrm{d}}$. Then, for times large compared to the advection time scale $\tau_{u}$, we obtain the following compact expressions for the effective dispersion coefficients,

$$
\begin{aligned}
\delta^{\mu v} D_{11}^{\mathrm{eff}}(t)= & \sqrt{\frac{\pi}{2}} \sigma_{\mu \mu}^{2} \sigma_{v v}^{2} u l \frac{\kappa^{2}}{a(\kappa)}\left[a(\kappa)-1+\left[\left(1+4 t / \tau_{\kappa}\right)^{\frac{1}{2}}-a(\kappa)\right]\right. \\
& \left.\times\left(1+4 t / \tau_{D_{2}}\right)^{-\frac{3}{2}} \prod_{n=3}^{d}\left(1+4 t / \tau_{D_{n}}\right)^{-\frac{1}{2}}\right], \\
\delta^{\mu v} D_{22}^{\mathrm{eff}}(t)= & \sqrt{\frac{\pi}{2}} \sigma_{\mu \mu}^{2} \sigma_{v v}^{2} u l a(\kappa)\left[1-\left(1+4 t / \tau_{\kappa}\right)^{-\frac{1}{2}} \prod_{n=2}^{d}\left(1+4 t / \tau_{D_{n}}\right)^{-\frac{1}{2}}\right],
\end{aligned}
$$

where we defined $a(\kappa)=\kappa / \sqrt{\kappa^{2}+1}$. The $\delta^{\mu v} D_{i i}^{\mathrm{eff}} \equiv 0$ for $i>2$ for symmetry reasons.

Note that $\delta^{\mu \nu} D_{11}^{\text {eff }} \leqslant 0$, while $\delta^{\mu \mu} D_{11}^{\text {eff }}+\delta^{\mu v} D_{11}^{\text {eff }} \geqslant 0$, for small $\sigma_{v v}^{2}$. For increasing $\sigma_{v v}^{2}$, this contribution can become negative, which, however, is a relic of the perturbation expansion in $\sigma_{v y}^{2}$, see Appendix A. Note that expansions in the fluctuations of the random fields can lead to nonconvergent series for some transient non-linear reactive

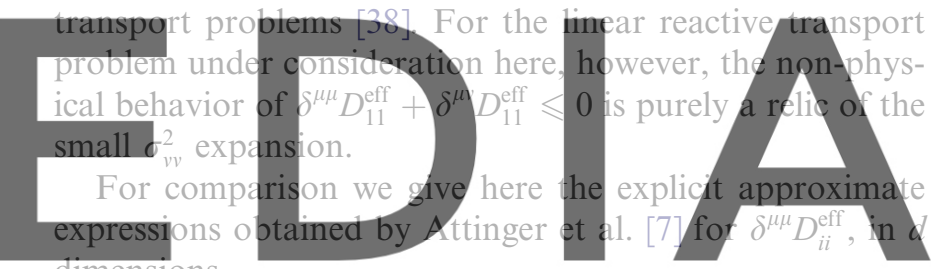

dimensions,

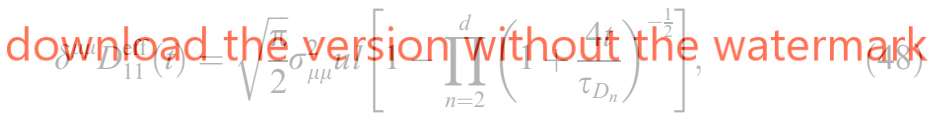

$\delta^{\mu \mu} D_{i i}^{\mathrm{eff}}(i)=0$.

Note, firstly, that the time evolution of $\delta^{\mu \mu} D_{11}^{\text {eff }}$ depends only on the transverse dispersion scale, and secondly that there is no macroscopic contribution to the transverse dispersion coefficient.

\section{Effective transport behavior}

It was shown by Attinger et al. [7] for transport under steady flow conditions that chemical medium heterogeneities change the behavior of the longitudinal dispersion coefficient in a quantitatively relevant way, whereas transverse solute spreading is only weakly influenced by the fluctuations of the retardation factor. As we saw at the end of the previous section, the effective transverse dispersion coefficient is in fact of the order of the local dispersion coefficient. For transport under a temporally fluctuating flow conditions, the behavior of transverse and longitudinal effective dispersion coefficients is different.

In the following, we investigate the asymptotic long time behavior and the temporal evolution of the effective disper- 
sion coefficients in $d=2$ dimensions. All results are normalized by $\sqrt{\frac{\pi}{2}} \sigma_{\mu \mu}^{2} \sigma_{v v}^{2} u l$. The behavior in $d=3$ dimensions is qualitatively similar.

\subsection{Asymptotic long time behavior}

We study here the asymptotic behavior of the contributions to the effective dispersion coefficients for isotropic local dispersion, $D_{11}=D_{22}=D$, as a function of the Kubo number $\kappa$. We define for the following,

$\lim _{t \rightarrow \infty} \delta^{\mu v} D_{11}^{\mathrm{eff}}(t)=\delta^{\mu v} D_{11}^{\infty}(\kappa)$,

$\lim _{t \rightarrow \infty} \delta^{\mu v} D_{22}^{\mathrm{eff}}(t)=\delta^{\mu v} D_{22}^{\infty}(\kappa)$.

Fig. 1 illustrates the asymptotic behavior of $\delta^{\mu v} D_{22}^{\infty}(\kappa)$ and $\delta^{\prime \prime \prime} D_{11}^{\infty}(\kappa)$. Both contributions to the longitudinal and transverse effective dispersion coefficients tend to zero in the limit $\kappa \rightarrow 0$. In this limit, the correlation time $\tau$ is much smaller than the advection time $\tau_{u}$, or equivalently, the Kubo length is much smaller than the correlation length, $l_{\kappa} \ll l$. Thus, for many correlation times, the medium looks quasi homogeneous to the transported solute and, as shown by Dentz and Carrera [29], there are no contribu-

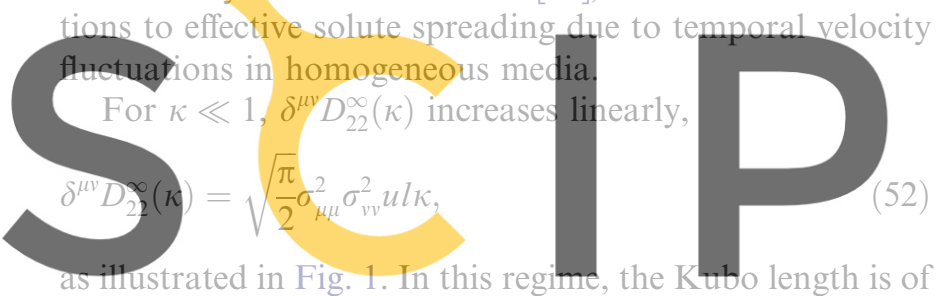

the order of the spatial correlation length, $l_{k} \lesssim l$, i.e., the

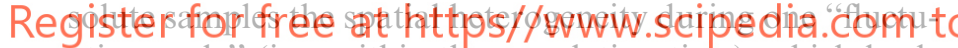

ation cycle" (i.e., within the correlation time), which leads

to enhanced spreading in transverse direction.

$\delta^{\mu v} D_{11}^{\infty}(\kappa)$, in contrast, decreases linearly in the same $\kappa$ interval,

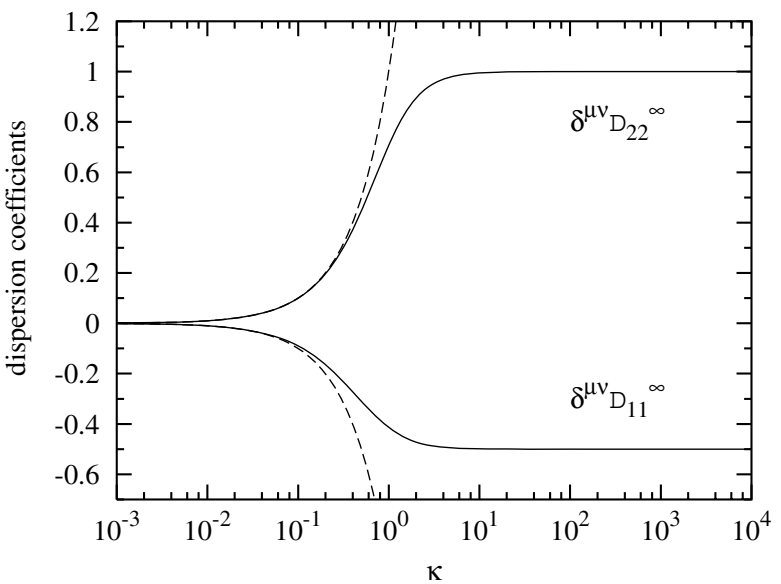

Fig. 1. Asymptotic behavior of the contributions to the effective dispersion coefficients due to chemical heterogeneity and temporal fluctuations in solid lines. The dashed lines describe the behavior of $\delta^{\mu v} D_{22}^{\infty}$ and $\delta^{\mu v} D_{11}^{\infty}$ according to (52) and (53), respectively. $\delta^{\mu v} D_{11}^{\infty}(\kappa)=-\frac{1}{2} \sqrt{\frac{\pi}{2}} \sigma_{\mu \mu}^{2} \sigma_{v v}^{2} u l \kappa$,

see Fig. 1. The contribution to the longitudinal effective dispersion coefficient decreases as the transverse coefficient is increasing. This will be discussed in the following.

In contrast to transport under steady flow conditions, where the disorder-induced contribution to effective transverse dispersion is of the order of local dispersion, here $\delta^{\mu v} D_{22}^{\infty}$ is macroscopic and dependent on the Kubo number $\kappa$. At the same time, the longitudinal dispersion coefficient decreases as $\delta^{\mu v} D_{11}^{\infty}$ is negative as shown in Fig. 1. The simultaneous increase of the transverse and decrease of the longitudinal effective dispersion coefficients can be seen as a consequence of self-organization of the system. Increased transverse spreading smoothes the concentration contrasts along directions normal to the mean flow, which in turn leads to a decrease of longitudinal effective spreading. A similar mechanism is known for the Taylor problem of dispersion in shear flow [37]. There, solute dispersion is enhanced as a consequence of the fact that the solute samples the transverse velocity contrast in the direction vertical to the mean flow. Vertical concentration contrasts, which

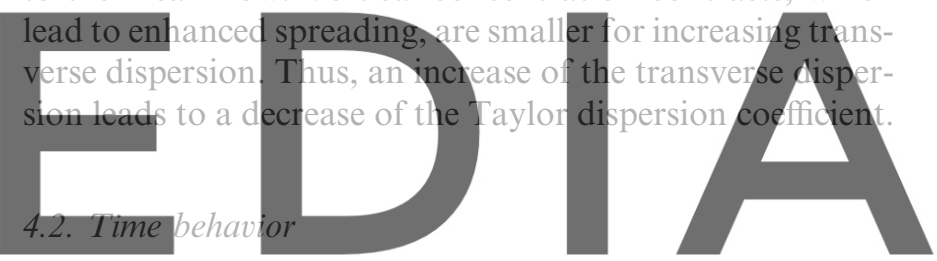

We study the time evolution of the contributions to the

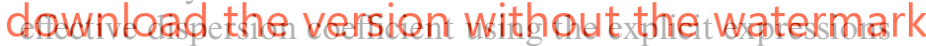
(46) and (47) for the longitudinal and transverse dispersion coefficients, respectively. We investigate different scenarios in order to study the different mechanisms which affect the behavior of the effective dispersion coefficients. At first we investigate an isotropic scenario for small and large Kubo numbers. Secondly, we investigate an anisotropic local dispersion scenario varying the longitudinal local dispersion coefficient.

\subsubsection{Isotropic scenario}

For the isotropic scenario, the inverse Peclet numbers $\epsilon_{i}$ and the dispersion time scales $\tau_{D_{i}}$ reduce to $\epsilon_{i}=\epsilon$ and $\tau_{D_{i}}=\tau_{D}$ for $i=1, \ldots, d$.

Figs. $2 \mathrm{a}$ and $2 \mathrm{~b}$ illustrate the time evolution of the contributions $\delta^{\mu v} D_{22}^{\text {eff }}$ to the transverse and $\delta^{\mu v} D_{11}^{\text {eff }}$ to the longitudinal effective dispersion coefficients in $d=2$ for $\kappa=10^{-1}$ and $\kappa=10$. The dispersion time scale is $\tau_{D}=10^{3} \tau_{u}$, i.e., the advection and dispersion time scales $\tau_{u}$ and $\tau_{D}$ are clearly separated.

For large values of $\kappa$, corresponding to $\tau_{\kappa} \gg \tau_{D}$, the Kubo scale $\tau_{\kappa}$ together with $\tau_{u}$ and $\tau_{D}$, separates three different time regimes: (i) the intermediate time regime $\tau_{u} \ll t \ll \tau_{D}$, (ii) the Kubo time regime $\tau_{D} \ll t \ll \tau_{\kappa}$, and (iii) the long-time regime $t \gg \tau_{\kappa}$. The Kubo scale sets a rel- 

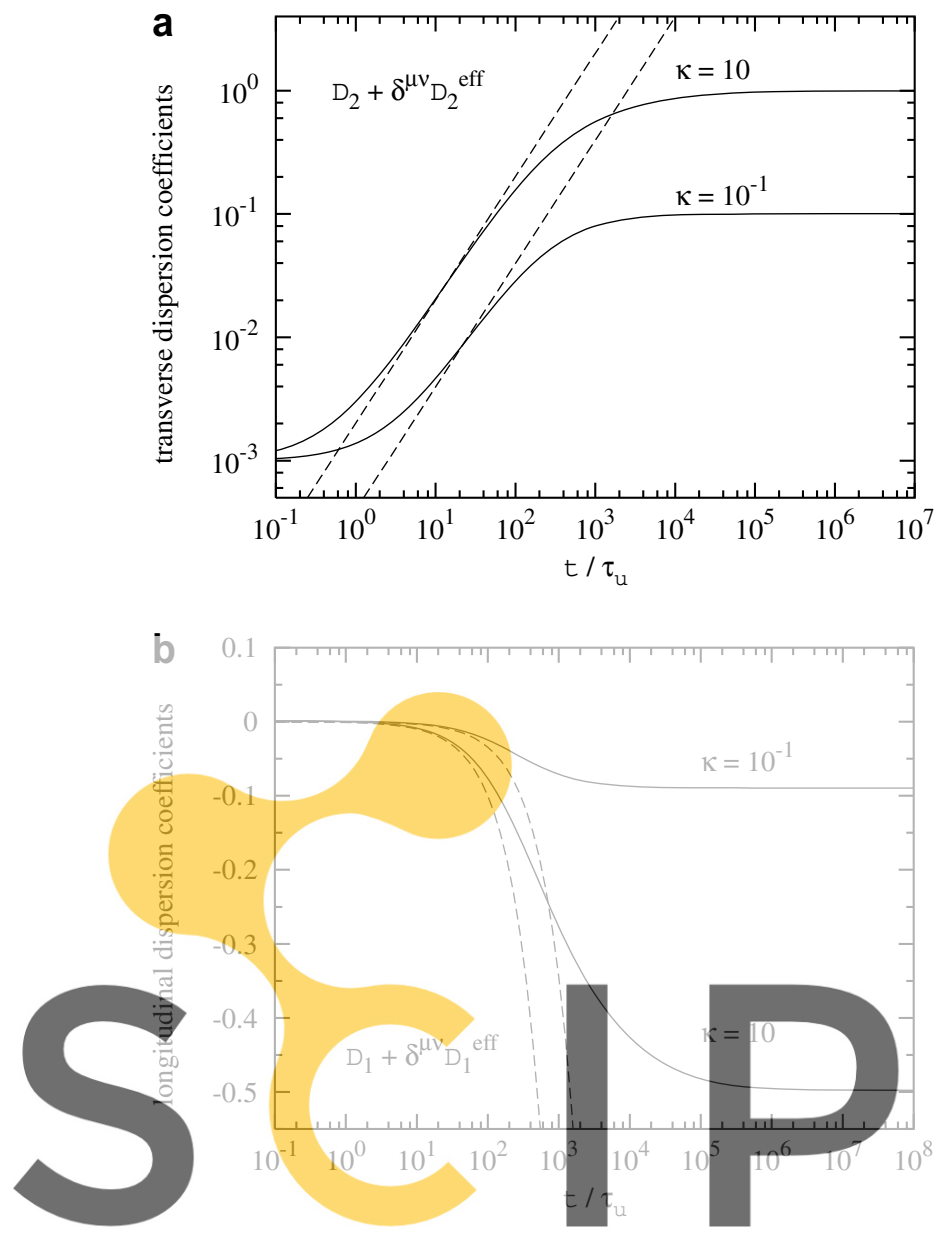

Fig. 2. Time behavior of the contributions to the (a) transverse effective

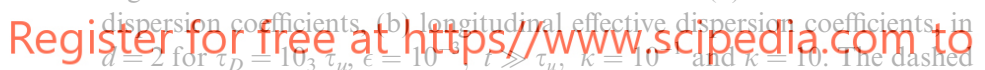
lines in (a) and (b) describe the linear approximations (54) and (55) of $\delta^{\mu v} D_{22}^{\text {eff }}$ and $\delta^{\mu v} D_{11}^{\text {eff }}$, respectively.

evant asymptotic time scale. Note that the solute has to be spread by local dispersion over at least one correlation length of the medium to "see" the chemical heterogeneity and it has to be spread over at least one Kubo length $l_{\kappa}$ to notice the influence of temporal flow fluctuations. Note that for small $\kappa, \tau_{\kappa} \approx \tau_{D}$, see definition (37), i.e., the Kubo scale is of importance for large Kubo numbers only. In order to illustrate this feature, we choose $\kappa=10^{-1}$ and $\kappa=10$.

\section{(i) Intermediate time regime $\tau_{u} \ll t \ll \tau_{D}$}

In this time regime, spatial heterogeneity is being activated as a macroscopic spreading mechanism and we observe a cross-over from local dispersive spreading and mixing to macroscopic heterogeneity induced effective dispersion. The contribution to the effective transverse dispersion coefficient, $\delta^{\mu v} D_{22}^{\text {eff }}$, both for $\kappa=10^{-1}$ and $\kappa=10$ evolves linearly with time,

$\delta^{\mu v} D_{22}^{\mathrm{eff}}(t)=\sqrt{2 \pi} \sigma_{\mu \mu}^{2} \sigma_{\mu \nu}^{2} u l a(\kappa) \frac{\left(\kappa^{2}+2\right)}{\left(\kappa^{2}+1\right)} \frac{t}{\tau_{D}}+\cdots$, where the dots denote subleading contributions. Contrary to the behavior observed under steady flow conditions, where transverse spreading is mainly given by local dispersion, here the transverse dispersion coefficient grows from the (microscopic) local scale dispersion coefficient to a macroscopic value. As shown in Fig. 2b, the contribution to the longitudinal coefficient decreases linearly according to,

$$
\delta^{\mu v} D_{11}^{\mathrm{eff}}(t)=-\sqrt{2 \pi} \sigma_{\mu \mu}^{2} \sigma_{\mu \nu}^{2} u l a(\kappa)\left[2+3 \kappa\left(\kappa-\sqrt{\kappa^{2}+1}\right)\right] \frac{t}{\tau_{D}}+\cdots,
$$

towards a negative macroscopic value and thus, longitudinal effective dispersion decreases as discussed in Section 4.1.

(ii) Kubo time regime $\tau_{D} \ll t \ll \tau_{\kappa}$
For $\kappa=10, \delta^{\mu \nu} D_{22}^{\text {eff }}$, see Fig. 2 a, evolves approximately according to $t^{-1 / 2}$, which is identical to the behavior observed for the longitudinal component under steady state conditions [7]. For $\kappa=10^{-1}$, i.e., $\tau_{\kappa} \approx \tau_{D}$ and the Kubo and long time regimes coincide. The contribution $\delta^{\mu v} D_{22}^{\text {eff }}$ evolves as $t^{-1}$ towards its asymptotic macroscopic value. The difference in the behaviors for $\kappa=10^{-1}$ and 10 can be well

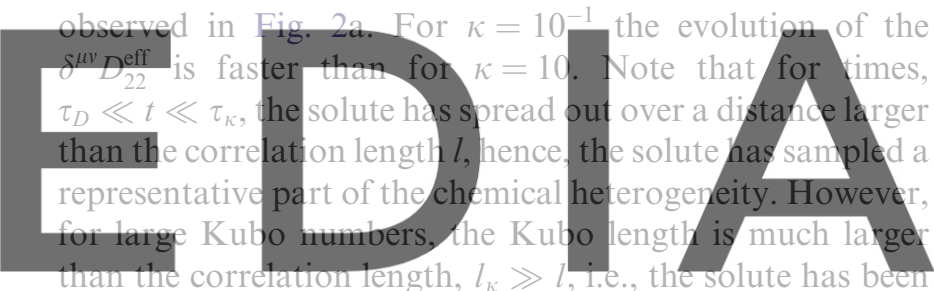

transported over more than one correlation length without

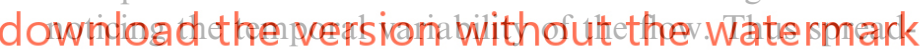
ing is dominated by the interaction of local dispersion and chemical heterogeneities, and the behavior of $\delta^{\mu \nu} D_{22}^{\text {eff }}$ is similar to the one observed for the longitudinal dispersion coefficient under steady flow conditions as in this time regime, $v(t)$ is approximately constant, i.e., there is an approximately constant transverse velocity component. For $\kappa=10^{-1}$, the solute has spread by local dispersion over distances larger than both, the correlation length and the Kubo length. Thus, both spatial heterogeneity as well as temporal flow fluctuations are activated as macroscopic spreading mechanisms. For this reason $\delta^{\mu v} D_{22}^{\text {eff }}$ evolves faster for $\kappa=10^{-1}$ than for $\kappa=10$. The same behavior can be observed in Fig. 2 for $\delta^{\mu v} D_{11}^{\text {eff }}$, which decreases faster for $\kappa=10^{-1}$ than for $\kappa=10$ for the reasons given above.

(iii) Long time regime $t \gg \tau_{\kappa}$

As pointed out above, for $\kappa=10^{-1}$, the Kubo and long time regimes coincide. For $\kappa=10$, the long time regime is set by the Kubo scale, see Figs. $2 \mathrm{a}$ and $2 \mathrm{~b}$. As pointed out above, only when the solute has been spread out over distances which are larger than both the correlation and the Kubo lengths, the interaction between chemical heterogeneity and temporal fluctuations are activated as macroscopic spreading mechanisms. As discussed above for $\kappa=10^{-1}$, here both $\delta^{\mu v} D_{22}^{\text {eff }}$ and $\delta^{\mu v} D_{11}^{\text {eff }}$ evolve towards their respective asymptotic values according to $t^{-1}$, i.e., faster 
than the contribution $\delta^{\mu \mu} D_{11}^{\text {eff }}$ in the absence of temporal flow fluctuations.

\subsubsection{Anisotropic scenario}

Here we study the temporal behavior of the effective dispersion coefficients for anisotropic local dispersion and isotropic disorder correlation.

Fig. 3a and $\mathrm{b}$ illustrate the time behavior of $\delta^{\mu v} D_{22}^{\text {eff }}$ and $\delta^{\mu v} D_{11}^{\text {eff }}$ for a fixed $\epsilon_{1}=10^{-1}$ and varying $\epsilon_{2}$ of $\epsilon_{2}=10^{-7}, 10^{-6}, 10^{-5}$ and $\epsilon_{2}=10^{-4}$, in $d=2$, for $\kappa=1$. The temporal behavior of $\delta^{\mu v} D_{2}^{\text {eff }}(t)$ is plotted only for the cases $\epsilon_{2}=10^{-7}$ and $\epsilon_{2}=10^{-4}$. For the values in between, the curves are basically identical.

For the isotropic scenario discussed in Section 4.2.1, $\tau_{k} \geqslant \tau_{D}$ and the asymptotic long time regime was defined by the Kubo time scale $\tau_{\kappa}$. For the anisotropic scenario under consideration here, the Kubo scale is smaller than the transverse dispersion time scale, $\tau_{\kappa} \ll \tau_{D_{2}}$. Thus, $\tau_{D_{2}}$ defines the relevant asymptotic time scale. These two time scales, along with the advection scale, define three time
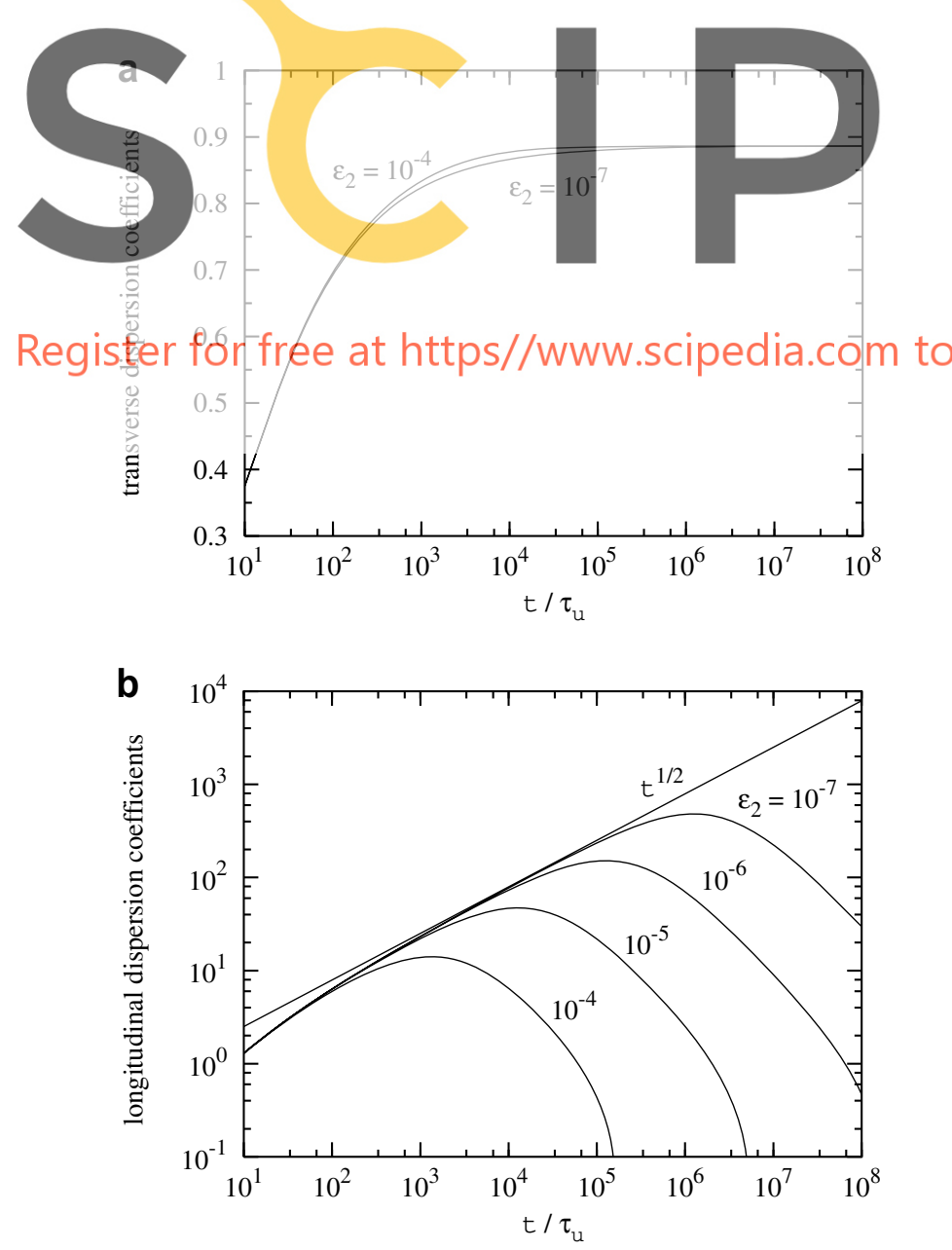

Fig. 3. Time behavior of (a) $\delta^{\mu v} D_{22}^{\text {eff }}(t)$, and (b) $\delta^{\mu v} D_{11}^{\text {eff }}$ (both scaled by $\left.\sqrt{\frac{\pi}{2}} \sigma_{\mu u}^{2} \sigma_{v v}^{2} u l\right)$ in $d=2$ for $\kappa=1$ and a fixed $\epsilon_{1}=10^{-1} ; \epsilon_{2}$ varies between $10^{-7}$ and $10^{-4}$. regimes which characterize the interaction of local dispersion, spatial heterogeneity and temporal fluctuations: (i) the Kubo time regime $\tau_{u} \ll t \ll \tau_{\kappa}$, (ii) the intermediate time regime $\tau_{\kappa} \ll t \ll \tau_{D_{2}}$, and (iii) the long-time regime $t \gg \tau_{D_{2}}$.

\section{(i) Kubo time regime $\tau_{u} \ll t \ll \tau_{\kappa}$.}

In this time regime, the solute has been transported by advection over a distance larger than the correlation length $l$ of the chemically heterogeneous medium. Solute has not been spread by local dispersion over distances larger than both the correlation length $l$ and the Kubo length $l_{\kappa}$. In this regime, temporal fluctuations and spatial heterogeneity are activated as relevant macroscopic spreading mechanism and we observe a cross-over from microscopic local dispersion to macroscopic disorder-induced spreading and mixing. The $\delta^{\mu \nu} D_{22}^{\text {eff }}$ and $\delta^{\mu \nu} D_{11}^{\text {eff }}$, see Figs. $3 \mathrm{a}$ and $3 \mathrm{~b}$, evolve linearly with time, as observed in the isotropic scenario.

(ii) Intermediate time regime $\tau_{\kappa} \ll t \ll \tau_{D_{2}}$.

Here, the solute has spread by longitudinal local dispersion over the Kubo length $l_{\kappa}$. In transverse direction, however, the solute has not yet sampled one spatial correlation

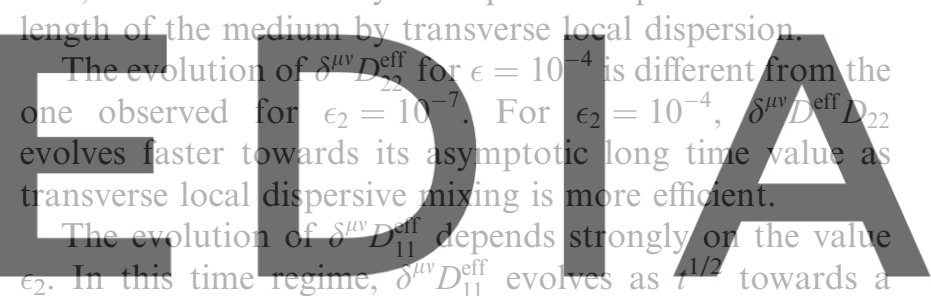

maximum, which it assumes for times of about $10^{-1} \tau_{D}$.

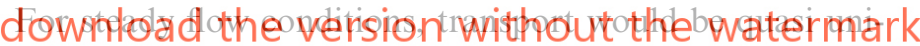
dimensional in this regime, as local transverse dispersion is subleading. In the presence of transverse flow fluctuations, however, there is vertical mass exchange. The transverse heterogeneity contrasts encountered by the solute leads then to the anomalous $t^{1 / 2}$ growth of $\delta^{\mu v} D_{11}^{\text {eff }}$. Note that local transverse dispersion, which smoothes out these vertical contrasts, is subleading. The anomalous diffusive behavior found here is similar to the one observed in stratified flow (e.g. $[39,40])$. The mechanisms are similar, but slightly different. While in the case of the stratified medium, local transverse dispersion is the vertical solute spreading and mixing mechanisms that leads, in interaction with the vertical velocity contrast, to the characteristic $t^{1 / 2}$ growth of the longitudinal effective dispersion coefficient, here transverse flow fluctuations cause vertical mass exchange. These mechanisms, local dispersion and transverse flow fluctuations, are different.

(iii) Long time regime $t \gg \tau_{D_{2}}$.

Here, the solute has spread over a correlation length of the medium by transverse local dispersion. Thus, spatial heterogeneity has been activated as a macroscopic spreading mechanism. The $\delta^{\mu v} D_{22}^{\text {eff }}$ converges towards its macroscopic asymptotic long time value. As a consequence of the increase in transverse effective dispersion, which leads to a smoothing out of the vertical heterogeneity contrast, 
$\delta^{\mu v} D_{11}^{\text {eff }}$, decreases towards its asymptotic long time value. The transverse dispersion time scale $\tau_{D_{2}}$ is a cut-off time scale for the anomalous diffusive behavior observed in the previous time regime.

\section{Summary and conclusions}

We investigate the effective transport of a reactive solute evolving from a point-like injection through a chemically heterogeneous medium. We focus on spatially fluctuating equilibrium sorption properties, which are characterized by a random retardation factor. The flow velocity is fluctuating in time with fluctuations transverse to the mean flow direction. In a stochastic modeling framework the spatial heterogeneity and temporal flow fluctuations are modeled by means of correlated spatial and temporal random fields.

The effective transport behavior is studied in terms of effective dispersion coefficients, which quantify the impact of spatio-temporal fluctuations on effective solute spreading and mixing. Using a first-order perturbation approach in the variances of the random processes, we derive explicit compact expressions for the time behavior of the transverse
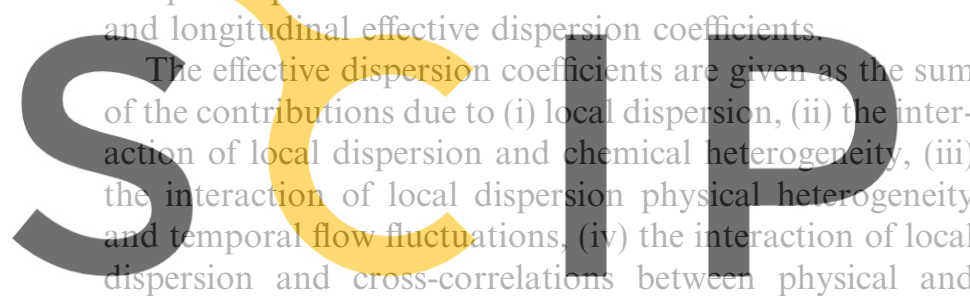

chemical heterogeneity, and (v) the interaction between

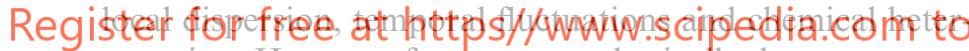
ogeneity. Here we focus on a physically homogeneous, chemically heterogeneous medium and study the latter contribution.

The time behavior of the effective dispersion coefficients are dominated by the following time scales: (i) the advection time scale $\tau_{u}=l / u$, (ii) the dispersion time scale $\tau_{D_{2}}=l / D_{22}$, which gives the activation time scale for chemical heterogeneities as a relevant macroscopic spreading mechanism, and (iii) the Kubo time scale $\tau_{\kappa}=\left(l^{2}+\right.$ $\left.l_{k}^{2}\right) / D_{11}$ which measures the time for the local dispersive spreading over a distance larger than both the correlation length $l$ and the Kubo length, $l_{\kappa}=u \tau$. Only after the time $\tau_{\kappa}$, the interaction between chemical heterogeneities and temporal flow fluctuations is activated as a macroscopic spreading mechanism.

Due to the interaction of temporal flow fluctuations and chemical heterogeneity, transverse effective dispersion evolves towards a macroscopic asymptotic value, which is in sharp contrast to the corresponding results for steady flow conditions, where no macroscopic contribution to transverse effective dispersion has been found.

In order to study the different spreading and mixing mechanisms and the interaction between them, we analyze two scenarios in $d=2$ spatial dimensions, (i) a completely isotropic scenario characterized by isotropic local disper- sion and disorder correlation, and (ii) a scenario characterized by an isotropic correlation structure and anisotropic local dispersion.

In the isotropic scenario, we distinguish three regimes, (i) the intermediate time regime $\tau_{u} \ll t \ll \tau_{D}$, (ii) the Kubo time regime $\tau_{D} \ll t \ll \tau_{\kappa}$ and (iii) the long time regime $t \gg \tau_{\kappa}$. In the intermediate regime, the plume size is still smaller than a disorder correlation length. Thus spatial heterogeneity has not been activated as an effective spreading mechanism. In the Kubo regime, the plume is larger than the disorder correlation length, but smaller than the Kubo-length. Thus, spatial heterogeneity is activated as a macroscopic spreading mechanism, time fluctuations are not. Only in the asymptotic regime, both spatial heterogeneity and temporal fluctuations are activated as effective spreading mechanisms.

For the anisotropic scenario we consider a different order of time scales, which implies (i) $\tau_{\kappa} \ll t \ll \tau_{D_{2}}$ and (ii) $t \gg \tau_{D_{2}}$. For $\tau_{\kappa} \ll t \ll \tau_{D_{2}}$, we observe superdiffusive behavior for the longitudinal effective dispersion coefficient. In this regime, transverse local dispersion is subleading and cannot smooth out the vertical concentration contrasts induced by the interaction of transverse redistri-

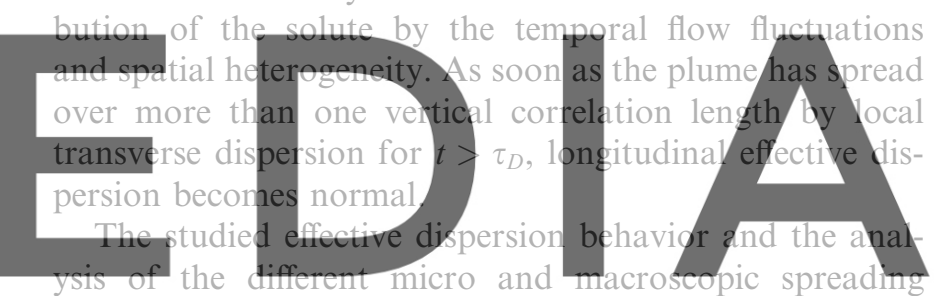
mechanisms and the interaction between them sheds some

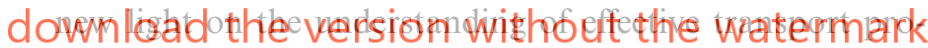
cesses in heterogeneous media. The observed increase of transverse dispersion due to temporal flow fluctuations can have important practical implications for the assessment of groundwater remediation based on hydraulic manipulation, as well as for the assessment and prediction of the migration of reactive contaminants in the context of performance assessment in nuclear waste management. The developed compact and simple expressions for the effective dispersion coefficients can be easily used for the quantification of effective solute spreading and mixing in such applications.

\section{Acknowledgments}

V.Z. gratefully acknowledges the financial support of the European Commission and the Departament d'Universitats, Recerca i Societat de la Informació de la Generalitat de Catalunya. M.D. gratefully acknowledges the support of the program 'Ramon y Cajal' of the Spanish Ministry of Education and Science (MEC). The finicial support by ENRESA and the EU through the IP FUNMIG (Contract No. 516514) and the MEC through the project MODEST (Contract No. CGL-2005-05171) is gratefully acknowledged. 


\section{Appendix A. Integral expressions}

Here we present the explicit integral expressions for the contributions to the effective transport velocity and the effective dispersion coefficients due to the interaction between temporal fluctuations of the flow conditions and chemical heterogeneity.

We derive approximate expressions for small inverse Peclet numbers. In order to keep the derivations as transparent as possible, the derived expressions are approximated successively, which means that, in the course of the derivations, we successively disregard subleading terms until we arrive at the consistent final result that represents the leading behavior in the limit of small inverse Peclet numbers.

Inserting expansion (28) for $\tilde{g}(\mathbf{k}, t)$ into $\overline{\ln [\tilde{g}(\mathbf{k}, t)]}$ and expanding the resulting expression up to first order in $\sigma_{\mu \mu}^{2}$, we obtain

$\overline{\ln [\tilde{g}(\mathbf{k}, t)]}=g_{0}(\mathbf{k}, t)+\sigma_{\mu \mu}^{2}\left[I_{1}(\mathbf{k}, t)+I_{2}(\mathbf{k}, t)\right]$

and thus for the effective center of mass velocity (31) and the effective dispersion coefficients (32),

$u_{i}^{\text {eff }}(t)=u \delta_{i 1}-\sigma_{\mu \mu}^{2} \frac{\mathrm{d}}{\mathrm{d} t} \mathrm{i} \frac{\partial}{\partial k_{i}}\left[\left\langle I_{1}(\mathbf{k}, t)\right\rangle-\frac{1}{2}\left\langle I_{2}(\mathbf{k}, t)\right\rangle\right]_{\mathbf{k}=\mathbf{0}}$,

$D_{i j}^{\mathrm{eff}}(t)=D_{i j}-\sigma_{\mu \mu}^{2} \frac{1}{2} \frac{\mathrm{d}}{\mathrm{d} t} \frac{\partial^{2}}{\partial k_{i} \partial k_{j}}\left[\left\langle I_{1}(\mathbf{k}, t)\right\rangle-\frac{1}{2}\left\langle I_{2}(\mathbf{k}, t)\right\rangle\right]_{\mathbf{k}=\mathbf{0}}$.

The auxiliary functions $I_{1}(\mathbf{k}, t)$ and $I_{2}(\mathbf{k}, t)$ are defined by

$$
\begin{aligned}
I_{1}(\mathbf{k}, t)= & \frac{1}{\tilde{g}_{0}(\mathbf{k}, t, 0)} \int_{k^{\prime}} \int_{-\infty}^{\infty} \mathrm{d} t^{\prime} \int_{-\infty}^{\infty} \mathrm{d} t^{\prime \prime} C^{\mu \mu}\left(\mathbf{k}^{\prime}\right) \tilde{g}_{0}\left(\mathbf{k}, t, t^{\prime}\right) \\
& \times \frac{\partial}{\partial t^{\prime}} \tilde{g}_{0}\left(\mathbf{k}-\mathbf{k}^{\prime}, t^{\prime}, t^{\prime \prime}\right) \frac{\partial}{\partial t^{\prime \prime}} \tilde{g}_{0}\left(\mathbf{k}, t^{\prime \prime}, 0\right), \\
I_{2}(\mathbf{k}, t)= & \frac{1}{\tilde{g}_{0}(\mathbf{k}, t, 0)^{2}} \int_{k^{\prime}} \int_{-\infty}^{\infty} \mathrm{d} t^{\prime} \int_{-\infty}^{\infty} \mathrm{d} t^{\prime \prime} C^{\mu \mu}\left(\mathbf{k}^{\prime}\right) \tilde{g}_{0}\left(\mathbf{k}, t, t^{\prime}\right) \\
& \times \frac{\partial}{\partial t^{\prime}} \tilde{g}_{0}\left(\mathbf{k}-\mathbf{k}^{\prime}, t^{\prime}, 0\right) \tilde{g}_{0}\left(\mathbf{k}, t, t^{\prime \prime}\right) \frac{\partial}{\partial t^{\prime \prime}} \tilde{g}_{0}\left(\mathbf{k}+\mathbf{k}^{\prime}, t^{\prime \prime}, 0\right),
\end{aligned}
$$

respectively. By partially integrating with respect to $t^{\prime}$ and $t^{\prime \prime}$, the internal time derivatives are shifted to the propagators that contain only external wave vectors $\mathbf{k}$. Evaluating the resulting time derivatives, we obtain for $I_{1}(\mathbf{k}, t)$ and $I_{2}(\mathbf{k}, t)$

$$
\begin{aligned}
& I_{1}(\mathbf{k}, t)=I_{11}(\mathbf{k}, t)+I_{12}(\mathbf{k}, t)+I_{13}(\mathbf{k}, t)+\cdots, \\
& I_{2}(\mathbf{k}, t)=I_{21}(\mathbf{k}, t)+2 I_{22}(\mathbf{k}, t)+\cdots,
\end{aligned}
$$

where the dots denote contributions which are small for small inverse Peclet numbers and contributions that are independent of $\mathbf{k}$. The auxiliary functions contributing to $I_{1}(\mathbf{k}, t)$ are defined by

$I_{11}(\mathbf{k}, t)=-\int_{k^{\prime}} \int_{0}^{t} \mathrm{~d} t^{\prime} \int_{0}^{t^{\prime}} \mathrm{d} t^{\prime \prime} \mathbf{k} \cdot \mathbf{u}\left(t^{\prime}\right) \mathbf{k} \cdot \mathbf{u}\left(t^{\prime \prime}\right) B_{1}\left(\mathbf{k}^{\prime}, t^{\prime}, t^{\prime \prime}\right)$,

$$
\begin{aligned}
& I_{12}(\mathbf{k}, t)=\int_{k^{\prime}} \int_{0}^{t} \mathrm{~d} t^{\prime} \mathbf{i k} \cdot \mathbf{u}\left(t^{\prime}\right) B_{1}\left(\mathbf{k}^{\prime}, t^{\prime}, 0\right), \\
& I_{13}(\mathbf{k}, t)=\int_{k^{\prime}} \int_{0}^{t} \mathrm{~d} t^{\prime} \mathbf{i k} \cdot \mathbf{u}\left(t^{\prime}\right) B_{1}\left(\mathbf{k}^{\prime}, t, t^{\prime}\right),
\end{aligned}
$$

where we defined

$B_{1}\left(\mathbf{k}^{\prime}, t_{1}, t_{2}\right)=\tilde{C}^{\mu \mu}\left(\mathbf{k}^{\prime}\right) \tilde{c}_{0}\left(-\mathbf{k}^{\prime}, t_{1}-t_{2}\right)\left[h\left(\mathbf{k}^{\prime}, t_{1}, t_{2}\right)+1\right]$

with

$h\left(\mathbf{k}^{\prime}, t_{1}, t_{2}\right)=\exp \left[-\mathrm{i} u \mathbf{k}^{\prime} \int_{t_{2}}^{t_{1}} \mathrm{~d} y \boldsymbol{v}(y)\right]-1$.

The auxiliary functions contributing to $I_{2}(\mathbf{k}, t)$ are given by $I_{21}(\mathbf{k}, t)=-\int_{k^{\prime}} \int_{0}^{t} \mathrm{~d} t^{\prime} \int_{0}^{t} \mathrm{~d} t^{\prime \prime} \mathbf{k} \cdot \mathbf{u}\left(t^{\prime}\right) \mathbf{k} \cdot \mathbf{u}\left(t^{\prime \prime}\right) B_{2}\left(\mathbf{k}^{\prime}, t^{\prime}, t^{\prime \prime}\right)$,

$I_{22}(\mathbf{k}, t)=\int_{k^{\prime}} \int_{0}^{t} \mathrm{~d} t^{\prime} \mathbf{i k} \cdot \mathbf{u}\left(t^{\prime}\right) B_{2}\left(\mathbf{k}^{\prime}, t, t^{\prime}\right)$,

where we defined

$$
B_{2}\left(\mathbf{k}^{\prime}, t_{1}, t_{2}\right)=\tilde{C}^{\mu \mu}\left(\mathbf{k}^{\prime}\right) \tilde{c}_{0}\left(-\mathbf{k}^{\prime}, t_{1}\right) \tilde{c}_{0}\left(\mathbf{k}^{\prime}, t_{2}\right)\left[h\left(\mathbf{k}^{\prime}, t_{1}, t_{2}\right)+1\right] .
$$

Note that the contributions $I_{11}(\mathbf{k}, t)$ and $I_{21}(\mathbf{k}, t)$ are of second order in $\mathbf{k}$ and contribute only to the effective dispersion coefficients, while $I_{12}(\mathbf{k}, t)$ and $I_{22}(\mathbf{k}, t)$ are linear in $\mathbf{k}$ and thus contribute only to the effective center of mass velocity.

The determination of the effective center of mass velocity and dispersion coefficients involves the following averages:

$$
\begin{aligned}
& \left\langle h\left(t_{1}, t_{2}\right)\right\rangle=\sigma_{v v}^{2} A\left(\mathbf{k}^{\prime}, t_{1}-t_{2}\right)+\cdots, \\
& \left\langle v_{l}\left(t_{3}\right) h\left(t_{1}, t_{2}\right)\right\rangle=\sigma_{v v}^{2} A_{l}\left(\mathbf{k}^{\prime}, t_{1}-t_{2}, t_{3}-t_{2}\right)+\cdots, \\
& \left\langle v_{l}\left(t_{3}\right) v_{\mathrm{m}}\left(t_{4}\right) h\left(t_{1}, t_{2}\right)\right\rangle=\sigma_{v v}^{2} C_{l m}^{v v}\left(t_{3}-t_{4}\right)+\cdots,
\end{aligned}
$$

where the dots denote contributions of the order of $\sigma_{v v}^{4}$. We defined the auxiliary functions

$$
\begin{aligned}
& A\left(\mathbf{k}^{\prime}, t\right)=-\frac{u^{2}}{2} \int_{0}^{t} \mathrm{~d} y \int_{0}^{t} \mathrm{~d} y^{\prime} k_{l}^{\prime} C_{l m}^{v v}\left(y-y^{\prime}\right) k_{\mathrm{m}}^{\prime}, \\
& A_{l}\left(\mathbf{k}^{\prime}, t_{1}, t_{2}\right)=-\mathrm{i} u \int_{0}^{t_{1}} \mathrm{~d} y C_{l m}^{v v}\left(t_{2}-y\right) k_{\mathrm{m}}^{\prime} .
\end{aligned}
$$

The average over $h\left(t_{1}, t_{2}\right)$ can be performed exactly for a Gaussian distributed $v(t)$. This yields,

$$
\left\langle h\left(t_{1}, t_{2}\right)\right\rangle=\exp \left[-\sigma_{v v}^{2} \frac{u^{2}}{2} \int_{t_{2}}^{t_{1}} \mathrm{~d} y \int_{t_{2}}^{t_{1}} \mathrm{~d} y^{\prime} k_{l}^{\prime} C_{l m}^{v v}\left(y-y^{\prime}\right) k_{\mathrm{m}}^{\prime}\right]-1 .
$$

Note that (A.20) is always positive, while the first order approximation of this expression in $\sigma_{v v}^{2}$ can be negative. Expression (A.20) gives the sum of $\delta^{\mu \mu} D_{11}^{\text {eff }}+\delta^{\mu \nu} D_{11}^{\text {eff }}$ and is always positive. The contribution (47) has been obtained by the first-order expansion of (A.20) and is 
strictly valid only for small variances at which $\delta^{\mu \mu} D_{11}^{\text {eff }}+\delta^{\mu v} D_{11}^{\text {eff }} \geqslant 0$.

In fact, the first-order approximation of (A.20) leads to unphysical negative values for the effective longitudinal dispersion coefficients for increasing variance.

Inserting (A.8), (A.9) and (A.13) into (A.2), expansion up to second order in the temporal fluctuations and subsequent average over the temporal random fields, we obtain for the effective center of mass velocity,

$u_{i}^{\text {eff }}(t)=u \delta_{i 1}+\delta^{\mu \mu} u_{i}^{\text {eff }}(t)+\delta^{\mu v} u_{i}^{\text {eff }}(t)$

with the contributions,

$$
\begin{aligned}
\delta^{\mu \mu} u_{i}^{\text {eff }}(t)= & u \delta_{i 1} \sigma_{\mu \mu}^{2} \int_{k^{\prime}} \tilde{C}^{\mu \mu}\left(\mathbf{k}^{\prime}\right) \tilde{c}_{0}\left(-\mathbf{k}^{\prime}, t\right)+\cdots, \\
\delta^{\mu v} u_{i}^{\text {eff }}(t)= & u \sigma_{\mu \mu}^{2} \sigma_{v v}^{2} \int_{k^{\prime}} \tilde{C}^{\mu \mu}\left(\mathbf{k}^{\prime}\right)\left[\delta_{i 1} A\left(\mathbf{k}^{\prime}, t\right)\right. \\
& \left.+A_{i}\left(\mathbf{k}^{\prime}, t, 0\right)\right] \tilde{c}_{0}\left(-\mathbf{k}^{\prime}, t\right)+\cdots
\end{aligned}
$$

where again the dots denote contributions that are small for small inverse Peclet numbers.

Correspondingly, we obtain for the effective dispersion coefficients by inserting (A.7) and (A.12) into (A.3),

$D_{i j}^{\mathrm{eff}}(t)=D_{i j}+\delta^{\mu \mu} D_{i j}^{\mathrm{eff}}(t)+\delta^{\mu v} D_{i j}^{\mathrm{eff}}(t)$,

where we defined,

$$
\begin{aligned}
\delta^{\mu \mu} D_{i j}^{\mathrm{eff}}(t)= & u^{2} \sigma_{\mu \mu}^{2} \delta_{i 1} \delta_{j 1} \int_{k^{\prime}} \int_{0}^{t} \mathrm{~d} t^{\prime} \tilde{C}^{\mu \mu}\left(\mathbf{k}^{\prime}\right) \tilde{c}_{0}\left(-\mathbf{k}^{\prime}, t^{\prime}\right) \\
& \times\left[1-\tilde{c}_{0}\left(\mathbf{k}^{\prime}, t\right)\right], \\
\delta^{\mu v} D_{i j}^{\mathrm{eff}}(t)= & u^{2} \sigma_{\mu \mu}^{2} \sigma_{v v}^{2} \int_{k^{\prime}} \int_{0}^{t} \mathrm{~d} t^{\prime} \tilde{C}^{\mu \mu}\left(\mathbf{k}^{\prime}\right)\left[\delta_{i 1} \delta_{j 1} A\left(\mathbf{k}^{\prime}, t^{\prime}\right)\right. \\
& \left.+\delta_{j 1} A_{i}\left(\mathbf{k}^{\prime}, t^{\prime}, 0\right)+\delta_{i 1} A_{j}\left(\mathbf{k}^{\prime}, t^{\prime}, 0\right)+C_{i j}^{v v}\left(t^{\prime}\right)\right] \\
& \times\left[\tilde{c}_{0}\left(-\mathbf{k}^{\prime}, t^{\prime}\right)-\tilde{c}_{0}\left(-\mathbf{k}^{\prime}, t\right) \tilde{c}_{0}\left(\mathbf{k}^{\prime}, t-t^{\prime}\right)\right] .
\end{aligned}
$$

We obtain the leading behavior of $\delta^{\mu v} u_{i}^{\text {eff }}(t)$ and $\delta^{\mu v} D_{i j}^{\text {eff }}(t)$ for small inverse Peclet numbers, (38) and (39) by inserting the expansions

$$
\begin{aligned}
\tilde{c}_{0}\left(-\mathbf{k}^{\prime}, t^{\prime}\right) & =\exp \left(-k_{j}^{\prime 2} l_{j}^{2} \epsilon_{j} t^{\prime} / \tau_{u}-\mathrm{i} k_{1}^{\prime} l_{1} t^{\prime} / \tau_{u}\right) \\
& =\exp \left(-\mathrm{i} k_{1}^{\prime} l_{1} t^{\prime} / \tau_{u}\right)+\cdots
\end{aligned}
$$

and

$$
\begin{aligned}
\tilde{c}_{0}\left(-\mathbf{k}^{\prime}, t\right) \tilde{c}_{0}\left(\mathbf{k}^{\prime}, t-t^{\prime}\right) & =\exp \left(-2{k_{j}^{\prime}}^{2} l_{j}^{2} t / \tau_{D_{j}}\right) \tilde{c}_{0}\left(\mathbf{k}^{\prime},-t^{\prime}\right) \\
& =\exp \left(-2{k_{j}^{\prime \prime}}^{\prime 2} l_{j}^{2} t / \tau_{D_{j}}-\mathrm{i} k_{1}^{\prime} l_{1} t^{\prime} / \tau_{u}\right)+\cdots
\end{aligned}
$$

into (A.23) and (A.26). The dots denote subleading contributions of the order of the inverse Peclet numbers.

\section{References}

[1] Chrysikopoulos C, Roberts KPKP. Analysis of one spatially variable retardation factor. Water Resour Res $1990 ; 26: 437-46$
[2] Bellin A, Rinaldo A, Bosma WJP, Zee SEATMVd, Rubin Y. Linear equilibrium adsorbing solute transport in physically and chemically heterogeneous posous formations 1. Analytical solutions. Water Resour Res 1993;29(12):4019-30.

[3] Miralles-Wilhelm F, Gelhar LW. Stochastic analysis of sorption macrokinetics in heterogeneous aquifers. Water Resour Res 1996;32(6):1541-9.

[4] Reichle R, Kinzelbach W. Effective parameters in heterogeneous and homogeneous transport models with kinetic sorption. Water Resour Res 1998;34(4):583-94.

[5] Metzger D, Kinzelbach H, Kinzelbach W. Asymptotic trasnport parameters in a heterogeneous porous medium: comparison of two ensemble-averaging procedures. Stochastic Env Res Risk Assessment 1999;13:396-415.

[6] Metzger D, Kinzelbach H, Kinzelbach W. Effective dispersion of a solute cloud in a chemically heterogeneous porous medium: comparison of two ensemble-averaging procedures. Water Resour Res 1996;32(11):3311-9.

[7] Attinger S, Dentz M, Kinzelbach H, Kinzelbach W. Temporal behaviour of a solute cloud in a chemically heterogeneous porous medium. J Fluid Mech 1999;386:77-104.

[8] Dentz M, Kinzelbach H, Attinger S, Kinzelbach W. Temporal behavior of a solute cloud in a heterogeneous porous medium: 1 . Point-like injection. Water Resour Res 2000;36(12):3591-604.

[9] Dentz M, Kinzelbach H, Attinger S, Kinzelbach W. Temporal behavior of a solute cloud in a heterogeneous porous medium: 2 . Spatially extended injection. Water Resour Res 2000;36(12):3605-14.

[10] Silliman SE, Simpson ES. Laboratory evidence of the scale effect in dispersion of solutes in porous media. Water Resour Res 1987;23:1667-73.

[11] Burr D, Naff R. Nonreactive and reactive solute transport in threedimensional heterogeneous porous media: mean displacement, plume spreading, and uncertainty. Water Resour Res 1994;30(3):791-815.

[12] Cortis A, Berkowitz B. Anomalous transport in "classical" soil and sand columns. Soil Sci Soc Am J 2004;68:1539-48.

[13] Fernández-Garcia D, Illangasekare TH, Rajaram H. Differences in the scale dependence of dispersivity and retardation factors estimated from forced-gradient and uniform flow tracer tests in three-dimensional physically and chemically heterogeneous porous media. Water Resour Res 2005;41(W03012). doi:10.1029/2004WR003125.

[14] Fernández-Garcia D, Rajaram H, Illangasekare TH. Assessment of the predictive capabilities of stochastic theories in a three-dimensional laboratory test aquifer: effective hydraulic conductivity and temporal moments of breakthrough curves. Water Resour Res 2005;41(W04002). doi:10.1029/2004WR003523.

[15] Lichtner PC, Tartakovsky DM. Upscaled effective rate constant for heterogeneous reactions. Stochastic Environ Res Risk Assessment (SERRA) 2003;17(6):419-29.

[16] Aucour AM, Tao FX, Moreira-Turcq P, Seyler P, Sheppard S, Benedetti MF. The Amazon River: behaviour of metals ( $\mathrm{Fe}, \mathrm{Al}, \mathrm{Mn}$ ) and dissolved organic matter in the initial mixing at the Rio Negro/ Solimoes confluence. Chem Geol 2003;197(1-4):271-85.

[17] Tonkin J, Balistrieri L, Murray J. Modeling metal removal onto natural particles formed during mixing of acid rock drainage with ambient surface water. Env Sci Tech 2002;36(3):484-92.

[18] Sanford W, Konikow L. Simulation of calcite dissolution and porosity changes in saltwater mixing zones in coastal aquifers. Water Resour Res 1989;25:655-67.

[19] Abarca E, Carrera J, Sanchez-Via X, Dentz M. Anisotropic dispersive henry problem. Adv Water Resour, in press. doi:10.1016/ j.advwatres.2006.08.005.

[20] Chu M, Kitanidis PK, McCarty PL. Modeling microbial reactions at the plume fringe subject to transverse mixing in porous media: when can the rates of microbial reaction be assumed to be instantaneous? Water Resour Res 2005;41:WR003495.

[21] Knutson CE, Werth CJ, Valocchi AJ. Pore-scale simulation of biomass growth along the transverse mixing zone of a model twodimensional porous medium. Water Resour Res 2005;41:W07007. 
[22] De Simoni M, Carrera J, Sanchez-Vila X, Guadagnini A. A procedure for the solution of multicomponent reactive transport problems. Water Resour Res 2005;41:WR004056.

[23] Kinzelbach W, Ackerer P. Modlisation du transport de contaminant dans un champ d'coulement non-permanent. Hydrolgologie 1986;2:197-205.

[24] Rehfeldt K, Gelhar L. Stochastic analysis of dispersion in usteady flow in heterogeneous aquifers. Water Resour Res 1992;28(8): 2085-99.

[25] Kabala Z, Sposito G. A stochastic model of reactive solute transport with time-varying velocity in a heterogeneous aquifer. Water Resour Res 1991;27(3):341-50.

[26] Zhang D, Neuman SP. Head and velocity covariances under quasisteady state flow and their effects on advective transport. Water Resour Res 1996;32:77-83.

[27] Dagan G, Bellin A, Rubin Y. Lagrangian analysis of transport in heterogeneous formations under transient flow conditions. Water Resour Res 1996;32(4):891-9.

[28] Cirpka OA. Effects of sorption on transverse mixing in transient flows. J Cont Hydrol 2005;78:207-29.

[29] Dentz M, Carrera J. Effective dispersion in temporally fluctuating flow through a heterogeneous medium. Phys Rev E 2003;68:036310.

[30] Cirpka OA, Attinger S. Effective dispersion in heterogeneous media under random transient flow conditions. Water Resour Res 2003;39(9):1257.
[31] Dentz M, Carrera J. Effective solute transport in temporally fluctuating flow through heterogeneous media. Water Resour Res 2005;41:W08414.

[32] Kitanidis PK. Prediction by the method of moments of transport in a heterogeneous formation. J Hydrology 1988;102:453-73.

[33] Kitanidis PK. The concept of the dilution index. Water Resour Res 1994;30:2011.

[34] Cirpka OA. Choice of dispersion coefficients in reactive transport calculations on smoothed fields. J Cont Hydrol 2002;58:261-82.

[35] Cirpka OA, Frind E, Helming R. Numerical simulation of biodegradiaton controlled by transverse mixing. J Cont Hydrol 1999;40(2):159-82.

[36] Abramowitz M, Stegun IA. Handbook of mathematical functions. New York: Dover Publications; 1972.

[37] Taylor GI. Dispersion of soluble matter in solvent flowing through a tube. Proc Roy Soc A 1953;219:186-203.

[38] Tartakovsky DM, Lichtner PC, Pawar RJ. PDF methods for reactive transport in porous media. Kovar K, Hrkal Z (editors), Conference on calibration and reliability in groundwater modelling (ModelCARE 2002), Prague, Czech Republic, June 17-20, IAHS-AISH Publication No. 277; 2003. p. 162-7.

[39] Matheron G, Marsily Gd. Is transport in porous media always diffusive? A counter-example. Water Resour Res 1980;16:901-17.

[40] Clincy M, Kinzelbach H. Stratified disordered media: exact solutions for transport parameters and their self-averaging properties. J Phys A 2001;34:7141-52. 